\title{
一种基于深度强化学习的 通信抗干扰智能决策方法
}

\author{
宋佰霖，许华，蒋否，饶宁
}

(空军工程大学 信息与导航学院, 陕西 西安 710077)

\begin{abstract}
摘 要: 为解决战场通信智能抗千扰决策问题,设计了一种基于深度强化学习的通信抗千扰决策方 法。该方法在 DQN 算法架构下引入经验回放和基于爬山策略 (PHC) 的动态 $\varepsilon$ 机制,提出动态 $\varepsilon$-DQN 智能决策算法,该算法能够根据决策网络状态更优地选择 $\varepsilon$ 值,提高收敛速度和决策成功率。在决策 过程中, 对所有通信频率是否存在千扰信号进行检测, 将结果作为千扰判别信息输入决策算法,使算 法可在无先验千扰信息条件下智能决策通信频率,在尽量保证通信不中断的前提下,有效躲避千扰。 实验结果表明,所提方法适应多种通信模型, 决策速度较快, 算法收敛后的平均成功率可达 $95 \%$ 以上, 较已有方法具有较大优势。
\end{abstract}

\section{关 键 词: 通信抗千扰; 智能决策; 深度强化学习 \\ 中图分类号:TN973 文献标志码: A \\ 文章编号: 1000-2758(2021) 03-0641-09}

随着战场电磁环境日趋复杂以及电子战技术的 快速发展 ${ }^{[1]}$, 军用无线通信受到的威胁越来越大, 提高通信抗干扰能力迫在眉睫, 要想尽可能保证通 信不受干扰,决策环节至关重要。

通信抗干扰过程是通信系统与干扰系统博弯的 过程,由于博亦论解决决策问题具有优势 ${ }^{[2]}$, 基于 博弯论的通信抗干扰决策和认知无线电研究取得一 定成果。这些研究主要基于前景理论 ${ }^{[3]}$ 、 Stackelberg 博弯 ${ }^{[4]}$ 和随机学习理论等 ${ }^{[5]}$ 方法, 从频 率、功率等 ${ }^{[6-7]}$ 角度人手, 通过构建通信与干扰间的 博弯模型, 计算出最优通信抗干扰策略。此类方法 的特点在于通信方需要获取干扰先验信息, 通过数 学推导得出结果。当干扰先验信息未知时, 此类方 法实现较为困难, 不足以应对干扰样式不断变化的 情况。

近年来, 随着对人工智能研究不断深人,一些基 于强化学习的通信抗干扰决策方法研究取得成果。 这些研究从功率分配 ${ }^{[8]}$ 、频率选择等 ${ }^{[9-10]}$ 角度人手, 综合运用模式识别 ${ }^{[10]}$ 、多智能体决策等 ${ }^{[11]}$ 领域知 识, 设计基于强化学习的方法进行决策。其中, 文献
[10]通过信号时频图识别干扰样式, 将频谱信息转 换为干扰样式信息, 根据不同干扰样式分别决策通 信频率; 文献 $[11]$ 提出一种基于 Q-Learning 算法的 多智能体协同抗干扰算法, 在扫频干扰的情况下抵 抗信道中的恶意干扰。此类决策方法最大优势在于 能够自动学习干扰信号的规律和特点, 自主决策出 当前状态下最优的抗干扰策略, 大幅度降低决策时 间, 提高决策准确率。

在抗干扰决策过程中, 改变通信频率是经常使 用的一种有效手段, 本文从通信频率选择角度人手, 将通信抗干扰决策与深度强化学习方法相结合, 提 出动态 $\varepsilon$-DQN 智能决策算法。利用能量检测法 ${ }^{[14]}$ 对各通信频率是否存在干扰信号进行检测, 得到当 前回合的干扰判别信息,输人决策算法,决策下一回 合的通信频率,并在干扰信号对准的同时改变通信 频率,有效躲避干扰。在不同通信场景下进行仿真 实验, 并与已有方法的决策效果进行对比, 验证本文 所提方法的有效性和适用性。 


\section{1 系统模型}

在通信场景中设置 1 个通信系统和 1 台干扰 机, 如图 1 所示, 通信系统由信号发射机、接收机, 干 扰检测模型和智能决策模型组成。

\section{1 信道传输模型}

在信号传输过程中, 用接收机的输人信干比 $\left(R_{\mathrm{SIN}}\right)$ 判定本次通信的被干扰程度,评估通信效果， $R_{\text {SIN }}$ 可用 $(1)$ 式表示。

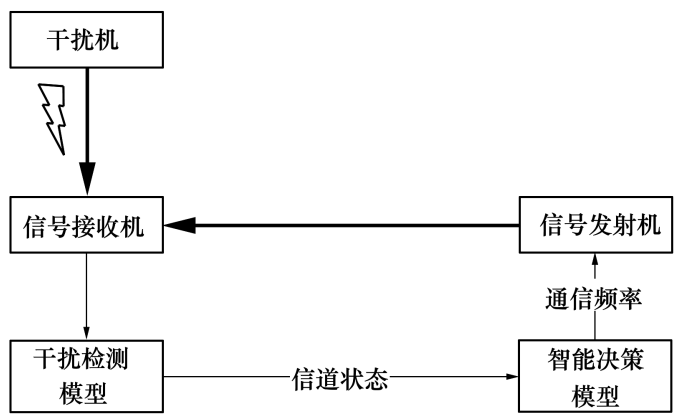

图 1 通信抗干扰智能决策方法的体系结构

$$
R_{\mathrm{SIN}}=\frac{P_{S} h_{S} L_{J}}{P_{J} h_{J} L_{S}}
$$

式中: $P_{S}$ 为信号发射机的发射功率; $P_{J}$ 为干扰机的 发射功率; $h_{S}$ 为信号发射天线增益与接收天线增益 之积; $h_{J}$ 为干扰机发射天线与信号接收天线增益之 积; $L_{S}$ 和 $L_{J}$ 分别为信号发射机和干扰机信号传输的 空间损耗,用 (2) 式表示, $r$ 为信号传播距离。

$$
L=32.5+20 \lg f+20 \lg r=20 \lg \frac{4 \pi r}{\lambda}=\left(\frac{4 \pi r}{\lambda}\right)^{2}
$$

为适应本文研究的问题模型, 所计算的 $R_{\mathrm{SIN}}$ 用 (3) 式表示 ${ }^{[15]}$,将(2) 式带人可得 (4) 式。

$$
\begin{gathered}
R_{\mathrm{SIN}}=\frac{P_{S} h_{S} L_{J}}{\sum_{j=1}^{J} \frac{P_{J}}{J} h_{J} \phi\left(f-f_{j}\right) L_{S}} \\
R_{\mathrm{SIN}}=\frac{P_{S} h_{S}}{\sum_{j=1}^{J} \frac{P_{J}}{J} h_{J} \phi\left(f-f_{j}\right)} \cdot\left(\frac{r_{J}}{r_{S}}\right)^{2}
\end{gathered}
$$

式中: $J$ 为干扰频率个数; $\phi(x)$ 为代表某频率是否 被干扰的指示值, 可用 (5) 式表示; $\sum_{j=1}^{J} \frac{P_{J}}{J} h_{J} \phi\left(f-f_{j}\right)$ 表示干扰信号在各个频率上的功率之和。

\section{2 通信模型}

$$
\phi\left(f-f_{j}\right)=\left\{\begin{array}{l}
1, f=f_{j} \\
0, f \neq f_{j}
\end{array}\right.
$$

为多角度验证本文所提出决策算法的有效性和 适应性,设置 2 个通信场景。通信时间以回合为基 准,每个回合进行 1 次通信。

- 场景 1: 每个回合仅选择 1 个频率进行通信, 共有 $N_{S}$ 个频率可供选择, 该频率上的信号功率为 $P_{S}^{[11]}$ 。若信干比超过國值 $q_{d}$, 认为当前回合正常 通信。

- 场景 2: 基于跳频通信体制, 每个回合在一个 频率集上进行通信, 每个频率集共有 $h$ 个频率, 共有 $N_{h}$ 个频率集可供选择, 每个频率上分配的信号功率 为 $P_{S} / h$ 。若总信干比大于阈值 $q_{h}$, 认为当前回合正 常通信。

\section{3 干扰模型}

根据上述 2 种不同通信场景, 设置 2 种有针对 性的干扰模型。

在对场景 1 的干扰模型中,设置扫频干扰、梳状 谱干扰和双频带扫频干扰 ${ }^{[11]} 3$ 种干扰样式, 每 20 个回合随机切换 1 次。

- 扫频干扰: 干扰机按照频率大小顺序顺次干 扰, 每回合干扰固定带宽 $B_{k}$, 带宽内分配的干扰功 率为 $P_{i}$ 。

- 梳状谱干扰: 干扰机每回合选择 $m_{j}$ 个干扰谱 组成梳状谱干扰, 每个干扰谱带宽为 $1 \mathrm{MHz}$, 带宽内 分配的干扰功率为 $P_{j} / m_{j}$ 。

- 双频带扫频干扰: 与扫频干扰类似,干扰机在 2 个频带上作相反顺序的扫频干扰, 每个干扰带宽 内分配的干扰功率为 $P_{i} / 2$ 。

对场景 2 的干扰模型设置 2 种干扰模式, “侦察 - 干扰” 模式和基于经验的干扰模式,2 种干扰样式 每 100 个回合随机改变 1 次。

在“侦察 - 干扰” 模式下,假设干扰机正在干扰 频率 $f_{0}$ 。从某时刻开始, 干扰机对当前通信频率 $f_{1}$ 进行侦察, 侦察时的干扰频率不变, 仍为 $f_{0}$ 。经过侦 察时间 $T$ 回合后,对侦察的频率 $f_{1}$ 实施干扰。初始 侦察时间为 $T_{j}$ 回合, 随着对抗回合数增加, 干扰机 逐渐熟悉当前通信体制，侦察时间逐渐减小，每增加 100 个回合, 侦察时间减少 1 个回合, 最短为 $T_{j \min }$ 回 合。考虑到侦察存在误差的可能性, 设置一个可信 概率 $p_{h}$, 有 $p_{h}$ 的可能干扰频率与通信频率完全相 符; 而有 $\left(1-p_{h}\right)$ 的可能存在部分频率侦察错误, 与 
通信频率不符。

在基于经验的干扰模式下, 干扰机选择最近 100 个回合里出现次数最多的通信频率进行干扰。

图 2 展示了这种模型下的通信和干扰状态, 横 轴为频率点数, 纵轴为时间回合数。绿色表示正常 通信的频率, 蓝色表示干扰成功的频率, 红色表示干 扰失败的频率。

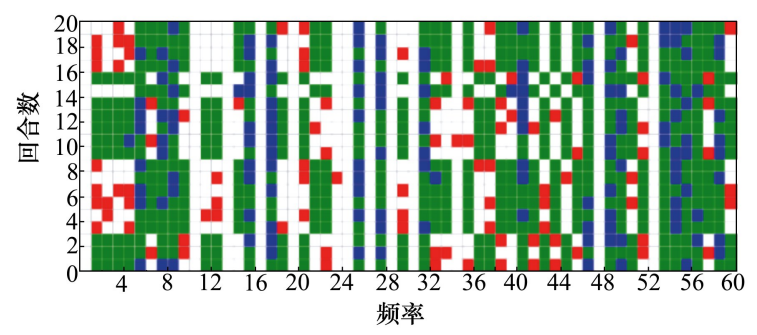

图 2 通信场景 2 的信道状态

\section{4 干扰检测模型}

根据信道内频率个数设置带通滤波器, 分别对 不同频率的信号进行滤波, 得到各个频率上的信号 功率 $P_{n}$, 设定一个检测模型 ${ }^{[14]}$

$$
\left\{\begin{array}{l}
H_{1}: y[n]=0 \\
H_{2}: y[n]=s[n] \\
H_{3}: y[n]=s[n]+j[n]
\end{array}\right.
$$

式中: $y[n]$ 表示某频率上的信号, 在不考虑外界环 境噪声的情况下, 它有 3 种可能的组成方式: 无信 号, 即 $y[n]=0$; 只有通信信号 $s[n]$; 通信信号 $s[n]$ 和干扰信号 $j[n]$ 共同组成。

计算每个频率的信号能量 $D(y)$, 用 (7) 式表 示 $^{[14]}$ 。设置门限值 $\lambda$, 对能量进行判别, 若高于 $\lambda$, 则认为该频率属于检测模型中的 $H_{3}$, 即存在干扰信 号; 否则属于检测模型中的 $H_{1}$ 或 $H_{2}$, 即不存在干扰 信号。将每个频率是否存在干扰信号的判别信息作 为信道状态, 输人智能决策模型。

\section{5 智能决策模型}

$$
D(y)=\sum_{n=1}^{N}\left|P_{n}\right|^{2}
$$

智能决策模型将接收到的信道状态输人动态 $\varepsilon-\mathrm{DQN}$ 智能决策算法, 根据当前回合的干扰频率, 给出下一回合通信频率, 输出决策模型。效能评估 模块根据信道状态和决策结果, 给出决策奖励值, 传 回算法,引导算法训练更新。

\section{2 动态 $\varepsilon-\mathrm{DQN}$ 智能决策算法}

\section{1 强化学习框架}

强化学习离不开智能体和环境 2 个基本条件, 智能体作为动作执行者, 与环境交互, 获取所需信 息,推动算法的训练更新。解决强化学习问题一般 有 5 个关键要素: 动作空间 $\boldsymbol{A}$, 状态空间 $\boldsymbol{S}$, 即时奖励 $r(s, a)$, 转移概率空间 $\boldsymbol{P}$ 和策略 $\boldsymbol{\pi}$ 。

当转移概率空间 $\boldsymbol{P}$ 未知时, 无法预测智能体与 环境的交互情况,这种强化学习被称为无模型的强 化学习,解决此类问题的传统算法是 $Q$-Learning 算 法。该算法建立一个 $Q$ 表,用表格的形式来存储每 个状态 - 动作价值 $Q(s, a), Q(s, a)$ 的计算过程可 用(8) 式表示。

$$
\begin{gathered}
Q(s, a)= \\
Q(s, a)+\alpha\left(r(s, a)+\gamma \max Q\left(s^{\prime}, a^{\prime}\right)-Q(s, a)\right)
\end{gathered}
$$

在算法训练阶段,通常将 $\varepsilon$-greedy 策略作为策 略 $\pi$ 进行训练更新。在该策略下,有 $1-\varepsilon$ 的概率个 体选择 $Q$ 值最大的动作, 有 $\varepsilon$ 的概率随机选择 动作。

$$
\pi(a \mid s)=\left\{\begin{array}{l}
\operatorname{argmax} Q(s, a), 0<x \leqslant 1-\varepsilon \\
\text { 随机选择, } 1-\varepsilon<x<1
\end{array}\right.
$$

当处理状态、动作空间较小的问题时, $Q-$ Learning 算法的效果较好, 但当处理状态、动作空间 较大的问题时, 搜索 $Q(s, a)$ 值会耗费大量时间, 导 致收玫速度降低, 且算法很多时候不能探索到所有 可能的动作, 收玫得到的策略 $\pi$ 并非全局最优, 此 时该算法便不再合适。

2013 年提出的 DQN 算法将图像或大数据集等 信息作为状态 $s$ 输人神经网络, 再输出动作空间 $\boldsymbol{A}$ 中各个动作对应的 $Q(s, a)$ 值, 代替了 $Q$ 表的运行机 制, 巧妙解决了上述问题。

\section{2 动态 $\varepsilon-\mathrm{DQN}$ 智能决策算法}

本文提出动态 $\varepsilon-\mathrm{DQN}$ 智能决策算法,运算流程 如图 3 所示。在强化学习框架下,将算法所需基本 元素定义如下:

1) 状态空间:分别将每个频率上的干扰判别信 息用 $S_{n}$ 表示, 若无干扰信号, $S_{n}=1$; 若有干扰信号, $S_{n}=-1$ 。将集合 $\boldsymbol{S}=\left[S_{1}, S_{2}, \cdots, S_{n}\right]$ 作为状态空间 输人算法。

2) 动作空间: 每个可选频率 $A_{n}$ 组成的集合 $\boldsymbol{A}$ 
作为动作空间。

3 ）即时奖励: 以当前回合信干比 $R_{\mathrm{SIN}}$ 为基础, 根据不同通信模型下的阈值 $q$ 设置奖励函数。当 $R_{\mathrm{SIN}}<q$ 时通信被成功干扰, $R_{\mathrm{SIN}}=0$; 当 $R_{\mathrm{SIN}} \geqslant q$ 时 通信正常, $R_{\mathrm{SIN}}=1$ 。为尽可能保证在同一频率上稳 定连续通信, 引人频率转换因子 $C_{\mathrm{tr}}{ }^{[15]}$, 若与上一回 合相比, 通信频率改变, 则 $C_{\mathrm{tr}}=0.6$, 否则 $C_{\mathrm{tr}}=0$, 即 时奖励 $r$ 可用 $(10)$ 式表示。

$$
r=\left\{\begin{array}{lc}
R_{\mathrm{SIN}}-C_{\mathrm{tr}}, & \text { 通信正常且改变频率 } \\
R_{\mathrm{SIN}}, & \text { 通信正常且未改变频率 } \\
0, & \text { 通信受扰且未改变频率 } \\
-C_{\mathrm{tr}}, & \text { 通信受扰且改变频率 }
\end{array}\right.
$$

传统的 $\varepsilon$-greedy 策略有固定的 $\varepsilon$ 概率随机选择 动作，使算法在任何回合的随机性相同。然而算法 在起始阶段和收玫阶段需要的随机性不同,固定的 $\varepsilon$ 值会导致算法向局部最优收敛且难以保持稳定的 收敛状态。本文基于 $\mathrm{PHC}$ 算法 ${ }^{[17]}$, 提出动态 $\varepsilon$ 策 略, 根据奖励值与回合数动态调整 $\varepsilon$ 值, 使算法收玫 到稳定的全局最优状态。PHC 算法在已知动作概 率 $\pi(s, a)$ 的条件下, 根据价值 $Q$ 动态调整概率, 尽 可能提高最大 $Q$ 值所对应动作的概率。

$$
\pi(s, a) \leftarrow \pi(s, a)+\Delta_{s a}
$$

式中

$$
\begin{aligned}
& \Delta_{s a}=\left\{\begin{array}{l}
-\delta_{s a}, a \neq \operatorname{argmax} Q(s, a) \\
\sum_{a \neq a^{\prime}} \delta_{s a^{\prime}}=\operatorname{argmax} Q\left(s, a^{\prime}\right)
\end{array}\right. \\
& \delta_{s a}=\min \left(\pi(s, a), \frac{\delta}{\left|A_{i}-1\right|}\right)
\end{aligned}
$$

当选择 $Q$ 值最大的动作时, 也相应增大该动作 的概率值; 当选择其他动作时, 则相应减小该动作的 概率值。本文结合 PHC 算法的动态调整方式, 将 $\varepsilon$ 初始值设置为 1 , 算法每迭代一个回合, $\varepsilon$ 值动态调 整 1 次, 直至 $\varepsilon$ 值为 0 。若 $r \leqslant 0$, 说明上一回合决策 失败, 则将 $\varepsilon$ 值减小, 增加其选择最优动作的概率, 减小策略的随机性,加速算法收敛; 若 $r>0$, 说明上 一回合决策成功, 则不改变 $\varepsilon$ 值, 使策略继续保持原 有随机性。动态 $\varepsilon$ 策略可用 $(15)$ 式表示, 在 $0 \sim 1$ 内随机生成数 $x$, 若 $x \leqslant 1-\varepsilon$, 则选择 $Q$ 值最大的动 作; 若 $x>1-\varepsilon$, 则随机选择动作。

$$
\begin{gathered}
\varepsilon=\left\{\begin{array}{l}
\varepsilon-t \times \varepsilon_{t}, r \leqslant 0 \\
\varepsilon, \quad r>0
\end{array}\right. \\
a=\left\{\begin{array}{l}
\operatorname{argmax} Q(s, a), 0<x \leqslant 1-\varepsilon \\
\text { 随机选择, } 1-\varepsilon<x<1
\end{array}\right.
\end{gathered}
$$

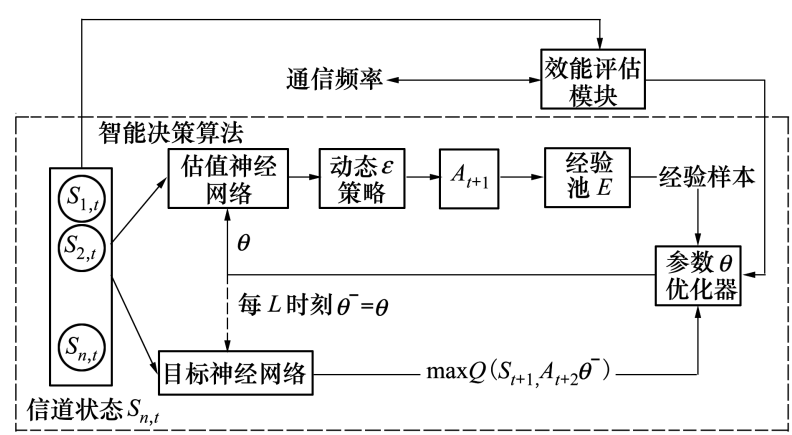

图 3 智能决策算法运算流程

这里引人一种经验回放机制 ${ }^{[18]}$,把最近回合的 经验 $e=\left\langle s, a, r, s^{\prime}>\right.$ 存人经验池 $\boldsymbol{E}$ 中, 在更新权重 值 $\theta$ 时,随机抽取部分经验样本进行更新, 以最大程 度破除相邻数据间的相关性并提高样本利用率。

算法中包含 2 个神经网络, 分别作为估值神经 网络和目标神经网络。估值神经网络负责给出当前 状态 $s$ 的估计价值 $Q\left(s, a ; \theta_{n}\right)$, 引导动作 $a$ 的选择。 目标神经网络负责给出目标价值 $Q\left(s^{\prime}, a^{\prime} ; \theta_{n}^{-}\right)$。其 中, $\theta_{n}$ 为在 $n$ 回合估值神经网络的权值参数, $\theta_{n}^{-}$为 在 $n$ 回合目标神经网络的权值参数。

定义误差函数 $L(\theta)$, 由 (16) 式表示。对参数 $\theta_{n}$ 作梯度下降计算, 以更新估值神经网络。每经过 $L$ 个回合, 将估值神经网络的权值参数赋给目标神 经网络,使 2 个网络参数相同,这样就不必实时更新 目标价值,减小了对目标价值选取的相关性。

$$
L\left(\theta_{n}\right)=\left[r+\gamma \max Q\left(s^{\prime}, a^{\prime} ; \theta_{n}^{-}\right)-Q\left(s, a ; \theta_{n}\right)\right]^{2}
$$

本文基于动态 $\varepsilon$-DQN 提出的智能决策算法, 设 置初始通信频率为 $A_{0}$, 算法根据当前回合的状态 $S_{t}$, 决策下一回合的通信频率 $A_{t+1}$ 。设置经验池大小 为 $N_{E}$, 选取的经验样本大小为 $N_{B}$ 。此时, 估计价值 和目标价值分别为 $Q\left(S_{t}, A_{t+1} ; \theta\right)$ 和 $Q\left(S_{t}, A_{t+1} ; \theta\right.$ -), (16) 式可写为

$$
\begin{gathered}
L(\theta)= \\
{\left[r\left(S_{t}, A_{t+1}\right)+\gamma \max Q\left(S_{t+1}, A_{t+2} ; \theta^{-}\right)-\right.} \\
\left.Q\left(S_{t}, A_{t+1} ; \theta\right)\right]^{2}
\end{gathered}
$$

神经网络参数更新的过程可分别用 (18)、(19) 式表 示。

$$
\begin{aligned}
& \theta \leftarrow \theta-\left[r\left(S_{t}, A_{t+1}\right)+\gamma \max Q\left(S_{t+1}, A_{t+2} ; \theta^{-}\right)-\right. \\
& \left.\quad Q\left(S_{t}, A_{t+1} ; \theta\right)\right] \nabla Q\left(S_{t}, A_{t+1} ; \theta\right) \\
& \theta^{-} \leftarrow \theta, t=n L(n=1,2, \cdots)
\end{aligned}
$$


算法 动态 $\varepsilon-\mathrm{DQN}$ 智能决策算法

1) 分别建立 2 个神经网络: 权值参数为 $\theta$ 的估 值神经网络和权值参数为 $\theta^{-}$的目标神经网络

2) 随机初始化权值参数 $\theta$, 令 $\theta^{-}=\theta$

3 ) 初始化选择通信频率作为初始动作 $A_{0}$, 设置 总回合数 $Z$

4) $t \leqslant Z$ 时,重复执行步骤 5) 13);

$5)$ 获得信道状态集合 $S_{t}$

6) 按照(14) 式计算 $\varepsilon$

7) 根据动态 $\varepsilon$ 策略选择下一回合通信频 率 $A_{t+1}$

$8)$ 按照通信频率 $A_{t+1}$ 进行信号传输

9) 获得即时奖励 $r\left(S_{t}, A_{t+1}\right)$

10) 获得下一回合的信道状态集合 $S_{t+1}$

11) 将经验样本 $e_{t}=\left\langle S_{t}, A_{t+1}, r\left(S_{t}, A_{t+1}\right), S_{t+1}>\right.$ 存人经验池 $E$ 中

12) 从经验池中随机选取经验样本 $N_{B}$ 个, 代人 到( 15) 式中更新估值神经网络的参数 $\theta$

13 ) 每 $L$ 个回合, 令 $\theta^{-}=\theta$, 使目标神经网络与 估值神经网络参数相同

14) $t>Z$ 时,执行完毕

算法中神经网络使用全连接网络, 激活函数设 置为 ReLU。将状态集合中的各个元素作为输人层 的各个元素, 其神经元个数为状态集合 $S_{t}$ 中的元素 个数 $N_{S}$; 神经元个数为动作集合 $\boldsymbol{A}_{t+1}$ 中的元素个数 $N_{A} ; n$ 个全连接层神经元个数均为 $N_{F}$, 总的神经元个 数为 $\left(N_{S}+N_{A}+n N_{F}\right)$ 。

该算法计算复杂度与神经网络有关, 输人层有 $N_{S}$ 个神经元, 那么第 1 个隐藏层有 $N_{S} N_{F}$ 个权重, 第 $n-1$ 个隐藏层有 $N_{F}^{2}$ 个隐藏层, 输出层共有 $N_{A} N_{F}$ 个 权重, 则整个神经网络共有 $N_{F}\left(N_{S}+(n-1) N_{F}+\right.$ $N_{A}$ ) 个权重。那么算法每一次迭代的复杂度可用 (20) 式表示。

$$
O\left(N_{F}\left(N_{S}+(n-1) N_{F}+N_{A}\right)\right)
$$

\section{3 实验与仿真分析}

本小节将干扰判别信息和干扰样式信息分别作 为算法输人, 对比动态 $\varepsilon-\mathrm{DQN}$ 算法、文献 [10] 中的 M-RL 决策算法和随机决策算法的决策效果, 表 1 为设定的模型参数。
表 1 模型参数设置

\begin{tabular}{cc}
\hline 参数 & 初始值 \\
\hline 信号发射机发射功率 $P_{s} / \mathrm{W}$ & 300 \\
干扰机发射功率 $P_{j} / \mathrm{W}$ & 9000 \\
通信信号增益 $h_{s}$ & 2 \\
干扰信号增益 $h_{j}$ & 2 \\
通信信号传播距离 $r_{s} / \mathrm{km}$ & 100 \\
干扰信号传播距离 $r_{j} / \mathrm{km}$ & 300 \\
衰减因子 $\gamma$ & 0.9 \\
神经网络学习率 $\alpha$ & 0.9 \\
经验池容量 $N_{E}$ & 500 \\
样本 B 的个数 $N_{\mathrm{B}}$ & 32 \\
$\varepsilon_{t}$ & 0.0004 \\
信道转换因子 $C_{\mathrm{tr}}$ & 0.6 \\
迭代总回合数 $Z$ & 10000 \\
\hline
\end{tabular}

\section{1 通信场景 1}

根据文献 $[10]$, 共有 5 个通信频率可供选择,

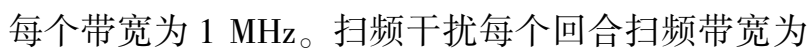
$200 \mathrm{kHz}$; 梳状谱干扰每个回合在第 1 、第 3 和第 5 个 频率设置共有 3 个干扰谱的干扰, 即 $m_{j}=3$, 信干比 的阈值 $q_{d}=0.9$ 。

设置估值神经网络和目标神经网络均包括输人 层、 1 个隐藏层和输出层。若干扰模型采用扫频干 扰,其扫频带宽小于通信信号带宽, 为体现干扰状 态, 以扫频干扰带宽为准设置输人层神经元数量, 为 $5 \times(1 \mathrm{MHz} / 200 \mathrm{kHz})=20$ 个, 其余 2 层神经元个数 分别为 16 和 5 。

图 4 所示为输人干扰判别信息时, 3 种决策算 法 10000 个回合下每百回合平均决策成功率对比。 动态 $\varepsilon-\mathrm{DQN}$ 算法在前 2000 个回合的决策成功率略 低于 M-RL 算法,这是由于算法采用动态 $\varepsilon$ 策略,收 玫前的随机性较强, 导致成功率相对较低。随着随 机性减弱,在 2500 个回合后,动态 $\varepsilon-\mathrm{DQN}$ 算法的决 策成功率逐渐收玫到 $100 \%$, 表现出优于其他 2 种算 法的决策效果。

图 5 所示为输人干扰样式信息时, 3 种算法的 平均决策成功率对比。与图 4 类似,动态 $\varepsilon-\mathrm{DQN}$ 算 法在 5000 回合左右达到收敛, 成功率稳定在 $98 \%$ 以上,但由于算法的随机性,在收玫前其决策成功率 低于 M-RL 算法。综合图 6 与图 7 可以看出, 动态 $\varepsilon-\mathrm{DQN}$ 算法在收敛后的决策成功率高于 M-RL 算 法,其决策效果更好。

图 6 所示为算法分别输人干扰判别信息和干扰 样式信息的平均决策成功率对比。从图中可以看 
出,由于输人干扰样式信息时,需要对不同干扰样式 所对应的决策网络进行训练,导致其收玫速度变慢; 相比于输人干扰样式信息,输人干扰判别信息的算 法收玫后决策成功率更高, 稳定在 $100 \%$ 。如果是
一种未知的干扰信号,将无法识别其干扰样式,而干 扰判别信息从能量角度人手检测, 不存在上述问题, 所以结合图 6 ,输人干扰判别信息的方法适应性更 强,决策效果更优。

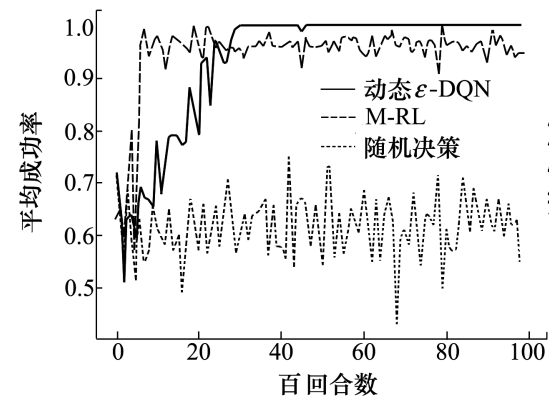

图 4 输人干扰判别信息的算 法平均决策成功率对比

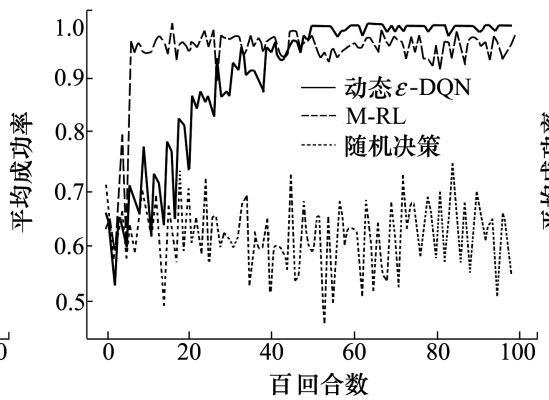

图 5 输人干扰样式信息的算 法平均决策成功率对比

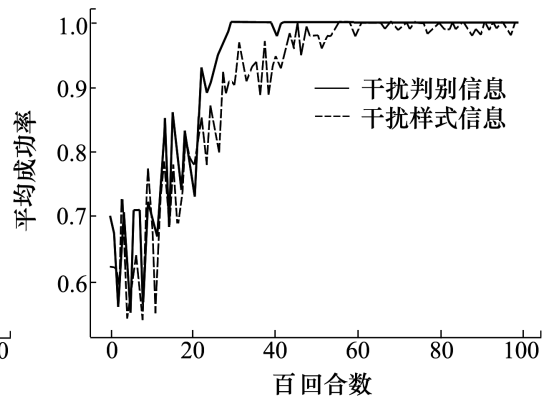

图 6 不同输人信息的算法 平均决策成功率对比
表 2 给出了输人不同信息时, 2 种智能决策算 法 10000 回合内的决策时间对比。无论输人何种 信息, 动态 $\varepsilon-\mathrm{DQN}$ 算法决策速度均更快, 且输人干 扰判别信息的算法决策速度最快, 仅需 $15.48 \mathrm{~s}$ 。

表 210000 回合决策时间对比

\begin{tabular}{ccc}
\hline 信息类型 & 动态 $\varepsilon$-DQN & M-RL \\
\hline 干扰判别 & 15.48 & 37.94 \\
干扰样式 & 36.93 & 42.85 \\
\hline
\end{tabular}

图 7 展示了分别采用动态 $\varepsilon$ 策略和不同 $\varepsilon$ 值的 $\varepsilon$-greedy 策略时, 算法的决策成功率对比。可以看 出随着 $\varepsilon$ 值不断增大, 算法采用 $\varepsilon$-greedy 策略收玫 后的平均成功率不断减小, 而采用动态 $\varepsilon$ 策略的算 法平均成功率稳定在 $100 \%$, 这说明相比于采用 $\varepsilon$ greedy 策略,动态 $\varepsilon$ 策略既提高了算法的收玫能力, 又提高了算法的决策成功率。

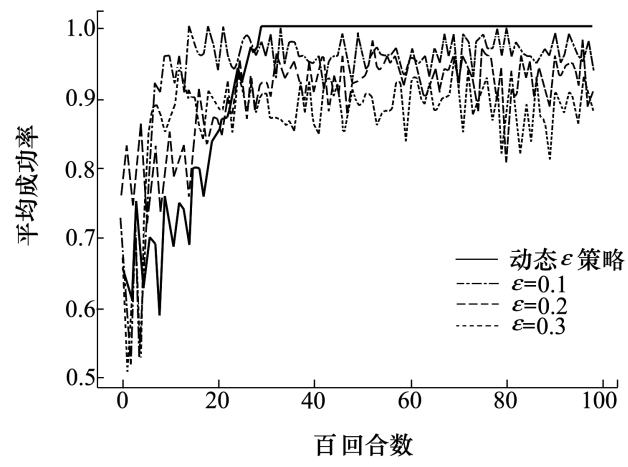

图 7 不同 $\varepsilon$ 值的算法决策成功率对比
由于动态 $\varepsilon-\mathrm{DQN}$ 算法采用了动态 $\varepsilon$ 策略,其随 机性随着迭代次数增加不断降低,算法收敛后的决 策成功率稳定在最优值上; 而 M-RL 算法由于一直 存在随机性, 其决策成功率在最优值下方不断波动, 这使得动态 $\varepsilon-\mathrm{DQN}$ 算法的决策成功率高于 M-RL 算法。

以输人干扰判别信息为例, 对 2 种智能决策算 法的计算复杂度进行比较。由 (20) 式可计算出当 前场景下动态 $\varepsilon-\mathrm{DQN}$ 算法 1 次迭代的计算复杂度 为 $O(400)$ 。由于 M-RL 算法是基于 $Q$-Learning 的 决策算法, 所以 M-RL 算法是遍历求解的, 可用 $O$ $(S A)$ 表示其计算复杂度 ${ }^{[19]}$ 。其中 $S$ 为输人算法的 状态总数, $A$ 为可供算法选择的动作总数,可计算得 到当前场景下算法的计算复杂度为 $O(205)$ 。

虽然动态 $\varepsilon-\mathrm{DQN}$ 算法的计算复杂度略高于 M$\mathrm{RL}$ 算法,但由于神经网络各层之间通过矩阵计算能 够降低算法的时间复杂度,所以动态 $\varepsilon-\mathrm{DQN}$ 算法的 决策速度依然较快。

\section{2 通信场景 2}

这一部分评估了动态 $\varepsilon-\mathrm{DQN}$ 算法在更复杂的 通信场景 2 下的性能。设置信道带宽为 $60 \mathrm{MHz}$, 共 有 60 个频率。有 5 个跳频频率集可供通信方选择, 每个频率集共 32 个频率, 每个回合干扰机可干扰 12 个频率。一般情况下,若频率集中有 $30 \%$ 的频率 被干扰, 则认为无法正常通信, 基于此设置阈值 $q_{h}=$ 0.33 ; 可信概率 $p_{h}=0.8$, 最初的侦察时间 $T_{j}=20$, 最 小侦察时间 $T_{j \min }=5$ 。

设置估值神经网络和目标神经网络均包括输人 
层、2 个隐藏层和输出层, 各层神经元数量分别为 $60,42,42$ 和 5 。

与通信场景 1 一样, 图 8 展示了输人干扰判别 信息时,3 种算法的每百回合平均决策成功率。可 以看出, 虽然 M-RL 算法收玫较快, 平均成功率在 $85 \%$ 上下波动, 但动态 $\varepsilon-\mathrm{DQN}$ 算法的平均决策成功 率更高, 在 3000 回合后平均成功率可达 $98 \%$ 以上。

图 9 所示为输人干扰样式信息时, 3 种算法的 平均决策成功率对比。动态 $\varepsilon-\mathrm{DQN}$ 算法在 4000 回
合左右达到收玫,成功率稳定在 $95 \%$ 以上, 综合图 8 与图 9 可以看出, 动态 $\varepsilon-\mathrm{DQN}$ 算法在收玫后的成功 率要高于 M-RL 算法, 其决策效果更好。

图 10 所示为算法分别输人干扰判别信息和干 扰样式信息时, 平均决策成功率对比。输人干扰样 式信息的算法收敛后的决策成功率在 90\% 100\% 间波动,而输人干扰判别信息的算法收敛速度更快 且成功率稳定在 $95 \%$ 以上, 决策效果更好。

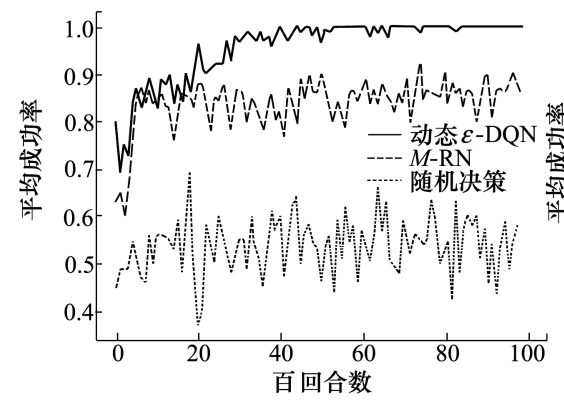

图 8 输人干扰判别信息的算 法平均决策成功率对比

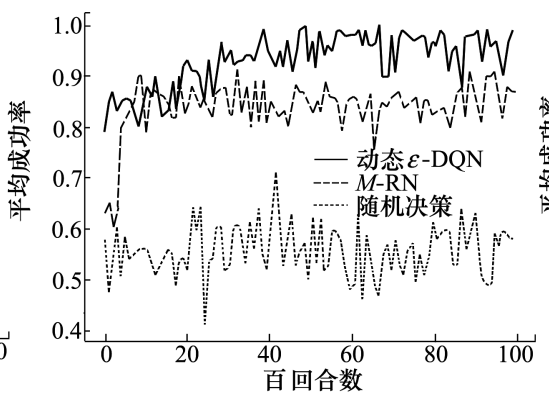

图 9 输人干扰样式信息的算 法平均决策成功率对比

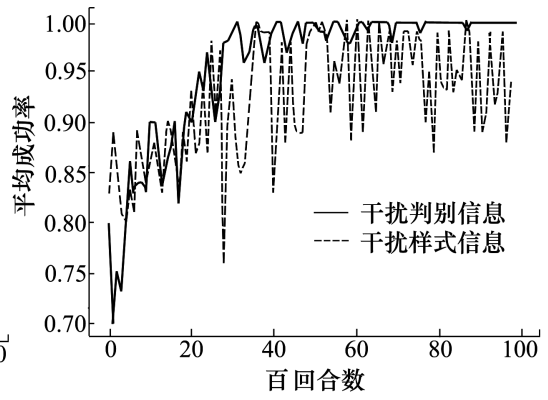

图 10 不同输人信息的算法 平均决策成功率对比
表 3 显示了动态 $\varepsilon-D Q N$ 算法和 M-RL 算法在通 信场景 2 下、输人不同信息时,10000 回合的决策时 间,与通信场景 1 相比, 2 种算法的决策速度均有所 减慢。但相比于 M-RL 算法, 动态 $\varepsilon-\mathrm{DQN}$ 算法的决 策速度快 $70 \%$ 以上, 且输人干扰判别信息时决策速 度最快, 仅需 $28.28 \mathrm{~s}$ 。

表 310000 回合决策时间对比

\begin{tabular}{ccc}
\hline 信息类型 & 动态 $\varepsilon$-DQN & M-RL \\
\hline 干扰判别 & 28.28 & 113.21 \\
干扰样式 & 33.38 & 136.14 \\
\hline
\end{tabular}

图 11 展示了采用动态 $\varepsilon$ 策略和不同 $\varepsilon$ 值的 $\varepsilon$ greedy 策略时, 算法决策成功率对比。与通信场景 1 类似, 可以看出随着 $\varepsilon$ 值不断增大, 收玫后的平均 成功率不断减小, 而采用动态 $\varepsilon$ 策略的算法平均成 功率在 3000 回合后, 可达 $95 \%$ 以上, 再次证明了动 态 $\varepsilon$ 策略较好的决策效果。

由于在该场景下干扰频率不是固定的, 会产生 大量不同的状态, 使得 M-RL 算法的训练不够充分, 在一些状态下决策出的通信频率不是最优的; 而动 态 $\varepsilon-\mathrm{DQN}$ 算法通过神经网络的拟合可以在任意干 扰状态下决策通信频率, 且保证准确率较高。同时, 采用了动态 $\varepsilon$ 策略的动态 $\varepsilon-\mathrm{DQN}$ 算法随着迭代进

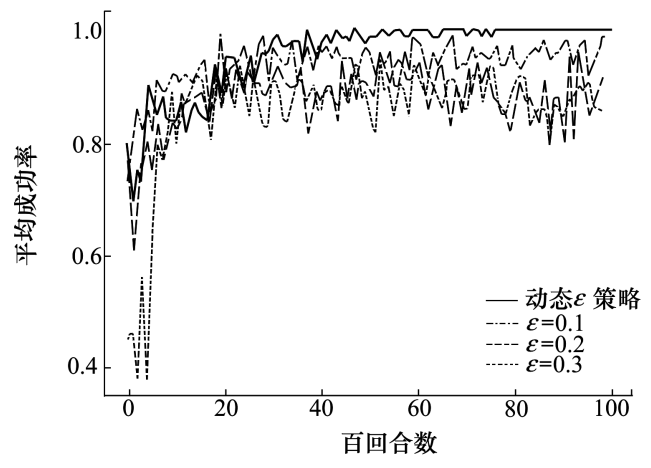

图 11 不同 $\varepsilon$ 值的算法决策成功率对比

行, 决策成功率将逐渐稳定在最优值上。

与场景 1 一样, 以输人干扰判别信息为例, 对 2 种智能决策算法的计算复杂度进行比较。由 (20) 式可计算出当前场景下动态 $\varepsilon-\mathrm{DQN}$ 算法的计算复 杂度为 $O(4494)$; 而干扰模型从 32 个频率中选择 12 个频率进行干扰, 可能产生的干扰状态最多有 $C_{32}^{16}$ 个, M-RL 算法的计算复杂度为 $O\left(5 C_{32}^{16}\right)$, 远超过 动态 $\varepsilon-\mathrm{DQN}$ 算法, 所以动态 $\varepsilon-\mathrm{DQN}$ 算法在决策速 度上的优势更为明显。

综合 2 个通信场景的算法结构和计算复杂度来 看, 模型越复杂, M-RL 算法的决策效果越低于动态 $\varepsilon-\mathrm{DQN}$ 算法。在复杂模型下, 只要将动态 $\varepsilon-\mathrm{DQN}$ 算 
法中的各类参数进行优化调整, 就能够解决当前的 决策问题, 虽然计算复杂度成倍增加, 但算法仍然能 够较为快速、准确地收敛到最优状态。

\section{4 结 论}

本文设计一种通信抗干扰智能决策方法, 该方 法基于 $\mathrm{DQN}$ 算法架构, 提出动态 $\varepsilon-\mathrm{DQN}$ 算法, 将当 前回合的干扰判别信息作为输人, 决策下一回合的 通信频率以躲避干扰。

综合 2 个典型通信场景下的仿真实验结果可分 析得出, 无论输人何种干扰信息, 动态 $\varepsilon$-DQN 算法 在收玫后的决策成功率均可达 $95 \%$ 以上, 当输人干 扰判别信息时, 决策成功率能够趋近 $100 \%$; 同时通
过对比 10000 回合的决策时间, 可以发现动态 $\varepsilon$ DQN 算法的决策速度远高于 M-RL 算法, 当模型越 复杂时,这种优势越显著,综合来看动态 $\varepsilon-\mathrm{DQN}$ 算 法的性能优于 M-RL 算法。对比输人不同干扰信息 的算法可以得出,输人干扰判别信息的算法无论决 策成功率还是决策速度均优于输人干扰样式信息的 算法; 由于干扰判别信息可直接通过干扰检测法获 得, 无需进行模式识别等复杂的处理步骤,该种信息 的获取更容易且适应性更强，所以将干扰判别信息 输人决策算法可获得事半功倍的效果。

综合决策性能指标来看,本文所提出的将干扰 判别信息输人动态 $\varepsilon-\mathrm{DQN}$ 算法的决策方法决策成 功率较高、速度较快, 决策效果较其他方法有较大 提升。

\section{参考文献:}

[1] VARDHAN S. Information jamming in electronic warfare: operational requirements and techniques[C]//International Conference on Electronics, 2015: 49-54

[2] XIAO L, CHEN T, LIU J, et al. Anti-jamming transmission stackelberg game with observation errors [J]. IEEE Communications Letters, 2015, 19(6): 949-952

[3] XIAO L, LIU J, LI Q, et al. User-centric view of jamming games in cognitive radio networks[ J ]. IEEE Trans on Information Forensics \& Security, 2015,10(12) : 2578-2590

[4] XIA T, PINY R, QINGH D, et al. Securing wireless transmission against reactive jamming: a stackelberg game framework[C] //IEEE Global Communications Conference, 2016: 1-6

[5] JIA L, XU Y, SUN, et al. A game-theoretic learning approach for anti-jamming dynamic spectrum access in dense wireless networks [J]. IEEE Trans on Vehicular Technology, 2018,68(2) : 1646-1656

[6] YANG D, XUE G, ZHANG J, et al. Coping with a smart jammer in wireless networks: a stackelberg game approach[J]. IEEE Trans on Wireless Communications, 2013, 12(8) : 4038-4047

[7] JIA L, YAO F, SUN, et al. A hierarchical learning solution for anti-jamming stackelberg game with discrete power strategies [J]. IEEE Wireless Communications Letters, 2017, 6(6) : 818-821

[8] NASIR Y S, GUO D. Multi-agent deep reinforcement learning for dynamic power allocation in wireless networks[ J]. IEEE Journal on Selected Areas in Communications, 2019, 37(10) : 2239-2250

[9] LIU X, XU Y, JIA L, et al. Anti-jamming communications using spectrum waterfall: a deep reinforcement learning approach [J]. IEEE Communications Letters, 2017, 22(5) : 998-1001

[10] LIU S, XU Y, CHEN X, et al. Pattern-aware intelligent anti-jamming communication: a sequential deep reinforcement learning $\operatorname{approach}[\mathrm{J}]$. IEEE Access, 2019, 7 : 169204-169216

[11] YAO F, JIA L. A collaborative multi-agent reinforcement learning anti-jamming algorithm in wireless networks[ J]. IEEE Wireless Communication Letters, 2019, 8(4) : 1024-1027

[12] MACHUZAK S, JAYAWEERA S K. Reinforcement learning based anti-jamming with wideband autonomous cognitive radios[C] // IEEE/CIC International Conference on Communications, 2016: 1-5

[13] AREF M A, JAYAWEERA S K, MACHUZAK S. Multi-agent reinforcement learning based cognitive anti-jamming[ C] //2017 IEEE Wireless Communications and Networking Conference( WCNC), 2017: 1-6

[14] LOPEZ-BENITEZ M, CASADEVALL F. Improved energy detection spectrum sensing for cognitive radio [ J]. IET Communications, 2012, 6(8) : 785-796 
[15] ZHANG Lin, TAN Junjie, LIANG Yingchang, et al. Deep reinforcement learning-based modulation and coding scheme selection in cognitive heterogeneous networks [J]. IEEE Trans on Wireless Communications, 2019, 18(6): 3281-3294

[16] HE Ying, ZHANG Zheng, YU Richard, et al. Deep-reinforcement-learning-based optimization for cache-enabled opportunistic interference alignment wireless networks [J]. IEEE Trans on Vehicular Technology, 2017, 66( 11) : 10433-10445

[17] YANG Bo, LEI, et al. A novel multi-agent decentralized win or learn fast policy hill-climbing with eligibility trace algorithm for smart generation control of interconnected complex power grids [ J ]. Elsevier Energy Conversion \& Management, 2015, 103: $82-93$

[18] MNIH V, KAVUKCUOGLU K, SILVER D. et al. Human-level control through deep reinforcement learning[J]. Nature, 2015, 518: 529-533

[19] CHI Jin, ZEYUAN Allenzhu, SEBASTIEN Bubeck, et al. Is Q-learning Provably Efficient? [ C] // 32nd International Conference on Neural Information Processing Systems, 2018: 4868-4878

\title{
An intelligent decision-making method for anti-jamming communication based on deep reinforcement learning
}

\author{
SONG Bailin, XU Hua, JIANG Lei, RAO Ning
}

(Information and Navigation College, Air Force Engineering University, Xi'an 710077, China)

\begin{abstract}
In order to solve the problem of intelligent anti-jamming decision-making in battlefield communication, this paper designs an intelligent decision-making method for communication anti-jamming based on deep reinforcement learning. Introducing experience replay and dynamic epsilon mechanism based on PHC under the framework of DQN algorithm, a dynamic epsilon-DQN intelligent decision-making method is proposed. The algorithm can better select the value of epsilon according to the state of the decision network and improve the convergence speed and decision success rate. During the decision-making process, the jamming signals of all communication frequencies are detected, and the results are input into the decision-making algorithm as jamming discriminant information, so that we can effectively avoid being jammed under the condition of no prior jamming information. The experimental results show that the proposed method adapts to various communication models, has a fast decision-making speed, and the average success rate of the convergent algorithm can reach more than 95\%, which has a great advantage over the existing decision-making methods.
\end{abstract}

Keywords : anti-jamming communication ; intelligent decision-making; deep reinforcement learning

引用格式: 宋伯霖, 许华, 蒋磊, 等. 一种基于深度强化学习的通信抗于扰智能决策方法 $[\mathrm{J}]$. 西北工业大学学报, 2021, 39 (3) : 641-649

SONG Bailin, XU Hua, JIANG Lei, et al. An intelligent decision-making method for anti-jamming communication based on deep reinforcement learning [J]. Journal of Northwestern Polytechnical University, 2021, 39(3): 641-649 (in Chinese) 